\section{Serviço de Atendimento Móvel de Urgência: Um observatório dos acidentes de transportes terrestre em nível local}

\section{Mobile Emergency Care Service: A survey of local land transportation accidents}

Amanda Priscila de Santana Cabral'

Wayner Vieira de Souza"

\section{Maria Luiza Carvalho de Lima"'}

' Núcleo de Estudos em Saúde Coletiva do Centro de Pesquisa Aggeu Magalhães da Fundação Oswaldo Cruz - PE.

"Laboratório de Métodos Quantitativos em Saúde do Núcleo de Estudos em Saúde Coletiva do Centro de Pesquisa Aggeu Magalhães da Fundação Oswaldo Cruz - PE.

"'Laboratório de Estudos em Violência e Saúde do Núcleo de Estudos em Saúde Coletiva do Centro de Pesquisa Aggeu Magalhães da Fundação Oswaldo Cruz $-\mathrm{PE}$.

\section{Resumo}

Conhecer a epidemiologia dos acidentes de transportes terrestres é fundamental para definir políticas de prevenção desse agravo e das mortes por ele causados. Objetivouse caracterizar o perfil epidemiológico das vítimas do trânsito e a distribuição dos atendimentos por acidentes de transporte a partir de técnica de análise espacial. Estudo descritivo, utilizou como fonte de dados o banco de atendimentos do Serviço de Atendimento Móvel de Urgência do município de Olinda, Pernambuco, entre julho de 2006 a junho de 2007. A distribuição geográfica das ocorrências foi analisada por meio do Índice de Moran. Pedestres, ocupantes de motocicleta e ciclistas concentraram $78 \%$ dos atendimentos; houve predomínio do sexo masculino (79\%) e da faixa etária 20-39 anos (65\%). Os finais de semana concentraram a maioria dos atendimentos $(56,1 \%$; $\left.\chi^{2}=123,7 ; p<0,001\right)$. Enquanto de segunda a quintas-feira $52 \%$ dos atendimentos se concentraram das $6 \mathrm{~h} 00$ às $17 \mathrm{~h} 59$, nos finais de semana, entre 18 h00 e 5h59, ocorreram $57 \%$ dos atendimentos. Motocicletas estão envolvidas em $68 \%$ dos acidentes e em 54\% dos atropelamentos. O Índice de Moran apontou regiões críticas, ratificando a importância do Sistema de Informação Geográfica e da análise espacial na vigilância dos acidentes de transportes terrestres. Os dados mostram o potencial dessa fonte de dados em contribuir no monitoramento permanente destes eventos ao apontar áreas geográficas e fatores associados ao maior risco de atendimentos no município. É pertinente a divulgação desses dados aos gestores, profissionais de saúde e o público em geral.

Palavras-Chave: Acidentes de transporte terrestre. Atendimento pré-hospitalar móvel. Perfil epidemiológico. Análise especial. Causas externas. Geoprocessamento. 


\section{Abstract}

Understanding the occurrence of land transportation accidents and describing the victims is fundamental for the definition of prevention and control policies regarding these events and the deaths they cause. The aim of the present study was to characterize the epidemiological profile of land transportation victims and the distribution of emergency care for land transportation accidents using spatial analysis. A descriptive study was carried out using the Mobile Emergency Care Service database of the city of Olinda (Pernambuco, Brazil) for occurrences between July 2006 and June 2007. The geographic distribution was analyzed using the Moran Index. Pedestrians, motorcyclists and bicyclists concentrated $78 \%$ of the emergency care; there was a predominance of male victims (79\%) and victims between 20 and 39 years of age (65\%). A greater concentration of occurrences was found on weekends $\left(56 \% ; \chi^{2}=123.7\right.$; $\mathrm{p}<0.001$ ). Between Monday and Thursday, $52 \%$ of occurrences were concentrated between 6 am and 5:59 pm; on weekends, $57 \%$ of the occurrences were concentrated between $6 \mathrm{pm}$ and 5:59 am. Motorcycles were involved in $68 \%$ of the accidents and $54 \%$ of run-over events. Spatial analysis using the Moran Index indicated regions of risk, thereby stressing the importance of the Geographic Information System and spatial analysis in the monitoring of land transportation accidents. The results demonstrate the potential of this data source to contribute for the permanent monitoring of these events by indicating geographic areas and factors associated with the risk of emergency care in the city. These data should be shown to administrators, healthcare professionals and the general public.

Keywords: Land transportation accidents. Mobile pre-hospital care. Epidemiological profile. Spatial analysis. External causes. Geoprocessing.

\section{Introdução}

Os Acidentes de Transporte Terrestre (ATT) são um fenômeno de abrangência mundial, relevante pela magnitude da mortalidade e do número de pessoas portadoras de sequelas decorrentes. São estimados, a cada ano, 1,2 milhões de óbitos por este evento, o que representa $12 \%$ das mortes do planeta, sendo a terceira causa mais frequente de mortes na faixa etária de 1 a 40 anos e a segunda causa na faixa etária de 5 a 29 anos ${ }^{1}$.

Pedestres e ciclistas são maioria dentre os mortos por ATT em países da África, Ásia e América Latina ${ }^{2}$. A rápida urbanização e a concomitante motorização nos países em desenvolvimento contribuíram para o crescimento dos ATT e isso não foi seguido por engenharia de trânsito apropriada e por programas de sensibilização, educação, prevenção de riscos e repressão das infrações ${ }^{3}$.

Sobre o setor saúde recai o maior ônus de todas as consequências dos acidentes, pois é ele quem cuida dos feridos, contabiliza as mortes e arca com os aspectos ligados às sequelas, não poucas vezes irreversíveis. As implicações dos ATT medidas em mortes, em demanda à atenção pré-hospitalar, hospitalar e de reabilitação, assim como as incapacidades geradas, os anos potenciais de vida perdidos, e o impacto nas famílias das vítimas e na sociedade em geral, têm levado instituições estrangeiras e nacionais a reconhecerem a sobrecarga que esses eventos produzem nos sistemas de saúde e o significativo custo social e econômico que representam ${ }^{4,5}$.

Estudos de perfil dos óbitos por ATT, baseados no Sistema de Informação sobre Mortalidade (SIM), têm fornecido importantes indicações quanto às características das vítimas, magnitude e transcendência, em diversas localidades ${ }^{6}$. Entretanto, a morte representa apenas a "ponta do iceberg" dos acidentes, pois o número de sobreviventes que demandam cuidados médicos, hospitalização e recursos de apoio diagnóstico pode ser bem maior ${ }^{7}$. Além disso, muitas declarações de óbito, apesar de informarem 
que se trata de morte por causa externa, deixam de detalhar o tipo específico da causa que provocou a lesão fatal, subestimando-as e levando a um aumento dos "eventos cuja intenção é indeterminada”.

A partir de 1998 a variável "tipo de acidente/violência” foi incorporada à autorização de internação hospitalar, tornando o Sistema de Internação Hospitalar (SIH) mais uma fonte de dados sobre ATT. No entanto, vale ressaltar que os dados correspondem somente aos pacientes internados e cujos gastos hospitalares foram custeados pelo SUS. Estima-se que a cobertura do SIH esteja em torno de $75 \%$, variando segundo o local e a complexidade dos procedimentos, podendo alcançar proporções mais elevadas em regiões mais dependentes do SUS ${ }^{9}$.

Visando complementar as informações existentes sobre causas externas, o Ministério da Saúde implantou em 2006 a Rede de Serviços Sentinela de Vigilância de Violências e Acidentes. Esta é formada pela vigilância de violências interpessoais em serviços de referência, com coleta universal e contínua e pela vigilância de acidentes e violências, cujos dados são coletados durante um mês a cada dois anos, em emergências hospitalares ${ }^{10}$.

O Departamento Nacional de Trânsito (Denatran), outra fonte de informações sobre ATT, propicia informações detalhadas sobre as circunstâncias dos acidentes como tipo de veículo envolvido, condições climáticas e da via no momento do acidente. Tem a vantagem de captar vítimas não fatais, possibilitando análise de tendências de morbidade; entretanto, não consegue captar todas as vítimas traumatizadas em acidentes ${ }^{8}$.

É consenso que, para a implantação e o desenvolvimento de políticas de prevenção dos ATT, deve-se estabelecer a vigilância, o monitoramento e a análise da situação para o conhecimento da magnitude das ocorrências, do perfil das vítimas, dos meios de transporte envolvidos e da localização das áreas de risco $^{6,7,11,12}$.

O Serviço de Atendimento Móvel de Urgência (Samu) é o componente pré- hospitalar móvel da Política Nacional de Atenção às Urgências ${ }^{13}$. Realiza atendimento de urgência e emergência nas residências, locais de trabalho e vias públicas, atendendo as demandas após chamada gratuita para o telefone $192^{14,15}$. Em 2008 o serviço funcionava em 1.269 municípios brasileiros, com 146 centrais de regulação e cobertura estimada de 101 milhões de pessoas ${ }^{16}$.

No município de Olinda, Pernambuco, o Samu foi implantado em fevereiro de 2006, integrando o Samu Metropolitano do Recife. Conta com médicos, enfermeiros, técnicos de enfermagem e condutores que se distribuem em quatro ambulâncias de suporte básico e uma ambulância de suporte avançado. A secretaria municipal de saúde implantou, de forma pioneira, o georreferenciamento de todos os atendimentos realizados e criou um banco de dados no software EpiInfo 3.3.2 com as variáveis do formulário de atendimento, possibilitando análises epidemiológicas a partir desta fonte. O georreferenciamento é possível uma vez que suas ambulâncias apresentam o aparelho Global Positioning System (GPS) e os condutores foram treinados a manuseá-lo ${ }^{11}$.

O presente trabalho objetiva descrever o perfil das vítimas por ATT e identificar as áreas de risco para essas ocorrências, a partir da demanda atendida pelo Samu em Olinda, possibilitando uma reflexão sobre o potencial dessa fonte como um observatório para os ATT em nível local.

\section{Métodos}

A área de estudo é o município de Olinda, localizado na região metropolitana de Recife, Pernambuco. No ano de 2006, contava com 387.496 habitantes ${ }^{17}$, distribuídos em um território de 40,8 $\mathrm{Km}^{2}$ e densidade demográfica de 9.490,4 habitantes/ $/ \mathrm{Km}^{2}{ }^{18}$.

Trata-se de um estudo descritivo, com utilização de dados secundários do banco de dados do Samu de Olinda, referentes ao total de ocorrências atendidas de 01 de julho de 2006 a 30 de junho de 2007.

Foram descritas a condição da vítima 
(se pedestre, ocupante de motocicleta, ciclista e ocupante de outros veículos), sexo, faixa etária, mês e veículo envolvido. Foram calculadas frequências relativas e taxas de incidência (TI; número de ocorrências/ população exposta *10 mil habitantes).

Para identificar diferença estatisticamente significante entre as proporções de atendimentos durante dias da semana e horários de atendimento foram calculados os riscos relativos. Utilizou-se o teste de qui-quadrado $\left(\chi^{2}\right)$, considerando como referências as quartas-feiras (para os dias de semana) e o período de 00h00 às 05h59 (para horário de atendimento), pois consideramos que esse dia e horários são os que sofrem menos influências de comportamentos humanos que podem levar à ocorrência de acidentes. Adotou-se intervalo com $95 \%$ de confiança e p-valor $<0,05$.

A população de referência foi a estimada pelo IBGE para o ano de $2006^{17}$, sendo 181.430 habitantes do sexo masculino e 206.066 do sexo feminino e, segundo faixa etária, 66.574 habitantes menores de 10 anos, 76.377 habitantes entre 10 e 19 anos, 134.703 habitantes entre 20 e 39 anos, 75.253 habitantes entre 40 e 59 anos e 34.589 habitantes entre 60 anos e mais, totalizando 387.496 as pessoas residentes no município.

Para identificação de áreas críticas, as ocorrências georreferenciadas foram agregadas em setores censitários, definidos pelo IBGE para o censo de 2001. Foram considerados o total de ocorrências atendidas por ATT e os atendimentos por ATT, tendo as seguintes categorias de vítimas: pedestres e ciclistas, motociclistas e ocupantes de veículo.

A análise da distribuição espacial dos atendimentos contou com 3 etapas. A primeira foi a identificação de áreas críticas e de transição, com a utilização do diagrama de espalhamento de Moran para comparar a dependência espacial de cada setor censitário. Nessa etapa, a área de estudo foi dividida nos quadrantes (Qn) onde Q1 (valores positivos, médias positivas) e Q2 (valores negativos, médias negativas) são pontos de associação espacial positiva, ou seja, setores censitários com número de atendimentos por ATT que possuem valores semelhantes aos seus vizinhos; Q3 (valores positivos, médias negativas) e Q4 (valores negativos, médias positivas) indicam pontos de associação espacial negativa, ou seja, setores censitários que possuem número de atendimentos por ATT distintos dos seus vizinhos. A representação visual dessa etapa se deu por meio do BoxMap ${ }^{19,20}$.

$\mathrm{Na}$ segunda etapa utilizou-se o Indicador Local de Associação Espacial (LISA), permitindo detectar regiões com correlação local significativamente diferente do resto dos dados, possível pela aplicação da estatística de autocorrelação espacial local. A avaliação de significância é feita comparando-se os valores adquiridos com uma série de valores obtidos por meio de permutações dos valores das áreas vizinhas. Os índices locais são classificados como não significantes e com significância de 95\%, $99 \%$ e 99,9\%. A representação visual desses dados se dá através do mapa denominado LisaMAP ${ }^{19,20}$.

A terceira etapa mescla as zonas que possuem relação espacial positiva, identificados pelo BoxMAP, com a significância espacial acima de 95\%, identificados pelo LisaMAP, gerando o MoranMAP. Consideraram-se áreas críticas aquelas formadas por setores censitários enquadrados na classe Q1 do MoranMap ${ }^{19,20}$.

Os softwares utilizados foram Microsoft Office Excell $2007^{\circledR}$, Epiinfo 6.0.4, Epiinfo 3.5.1 e TerraView 3.2.0.

O projeto de pesquisa foi submetido e aprovado pelo Comitê de Ética em Pesquisa do Centro de Pesquisas Aggeu Magalhães/Fiocruz, sob registro no CAEE No 0008.0.095.000-08.

\section{Resultados}

\section{Perfil epidemiológico das vítimas e ocorrências atendidas pelo Samu 192 Olinda}

O Samu 192 Olinda atendeu 1032 indivíduos por ATT entre 01 de julho de 2006 e 
30 de junho de 2007. O sexo masculino foi o mais atendido $(795 ; 78,9 \%)$ e também o mais acometido (43,8/10 mil habitantes). Pedestres e ciclistas responderam por 602 atendimentos $(58,3 \%)$ e ocupantes de motocicleta por 199 (19,3\%) (Tabela 1).

Quanto à faixa etária, 640 (65,0\%) atendimentos se destinaram a pacientes entre 20 e 39 anos, sendo em $47,8 \%$ dos casos na condição de pedestre e $24,4 \%$ como ocupantes de motocicleta (Tabela 1).

Os meses de maior número de atendimentos foram fevereiro/2007 (112; 10,2\%), abril/2007 (99; 9,6\%) e outubro/2006 (98; $9,5 \%)$. Nos finais de semana foram realizados 581 atendimentos (56,1\%). Detectouse diferença estatisticamente significante entre as proporções do total de atendimentos segundo dias de semana $\left(\chi^{2}=123,7\right.$; $\mathrm{p}<0,001)$, sendo os dias de maior risco de atendimentos o sábado $(\mathrm{RR}=2,4)$ e o domingo ( $\mathrm{RR}=2,5)$ (Tabela 2).

Houve concentração de atendimentos entre $18 \mathrm{~h} 00$ e 23h59, com diferença estatisticamente significante entre a proporção de atendimentos segundo horário do acidente, tanto de segunda a quinta-feira $\left(\chi^{2}=97,5\right.$; $\mathrm{p}<0,01)$, como nos fins de semana $\left(\chi^{2}=106\right.$; $\mathrm{p}<0,01)$. Tomando como referência o horário entre 00h00 às 05h59, de segunda-feira a quinta-feira, a incidência de atendimentos por ATT entre $18 \mathrm{~h} 00$ e $23 \mathrm{~h} 59$ foi 4 vezes maior, enquanto nos finais de semana foi maior em 2,5 vezes (Tabela 2 ).

Também se constatou diferença estatisticamente significante entre a distribuição horária dos acidentes, comparando segunda-feira a quinta-feira e o fim de semana $\left(\chi^{2}=13,81 ; p=0,003\right)$. Observou-se que $52 \%$ dos atendimentos de segunda a quinta feira se concentraram entre 06h00 e 17h59, enquanto nos finais de semana $57 \%$ das ocorrências se deram das $18 \mathrm{~h} 00$ às $05 \mathrm{~h} 59$ (Tabela 2).

Dentre os tipos de veículos envolvidos no cenário do acidente, destacaram-se as motocicletas (592; 57,4\%), seguidas dos carros de passeio $(165 ; 16,0 \%)$ e dos ônibus (100; 9,7\%). Sabendo que os pedestres representam o maior volume de atendimentos, investigou-se a proporção de veículos envolvidos, considerando essa condição de

Tabela 1 - Ocorrências atendidas pelo SAMU 192 segundo idade, sexo e condição da vítima. Olinda, PE - Julho de 2006 a Junho de 2007.

Table 1 - Occurrences involving the Mobile Emergency Care Service according to age, gender and condition of victim; Olinda, PE, Brazil; July 2006 to June 2007.

\begin{tabular}{|c|c|c|c|c|c|c|c|c|c|c|c|c|c|c|c|c|c|c|}
\hline & & \multicolumn{3}{|c|}{ Pedestre } & \multicolumn{3}{|c|}{ Ciclista } & \multicolumn{3}{|c|}{$\begin{array}{c}\text { Ocupante de } \\
\text { motocicleta }\end{array}$} & \multicolumn{3}{|c|}{$\begin{array}{c}\text { Ocupante de outros } \\
\text { veículos }\end{array}$} & \multicolumn{2}{|c|}{ Ignorado } & \multicolumn{3}{|c|}{ Total } \\
\hline & & $\mathrm{N}$ & $\%$ & $\mathrm{TI}^{(1)}$ & $\mathrm{N}$ & $\%$ & $\mathrm{TI}^{(1)}$ & $\mathrm{N}$ & $\%$ & $\mathrm{~T}^{(1)}$ & $\mathrm{N}$ & $\%$ & $\mathrm{Tl}^{(1)}$ & $\mathrm{N}$ & $\%$ & $\mathrm{~N}$ & $\%$ & $\mathrm{TI}^{(1)}$ \\
\hline \multirow{5}{*}{$\stackrel{\substack{x \\
\sim}}{\mathfrak{N}}$} & Masculino & 407 & 51.2 & 22,4 & 51 & 6.4 & 2,8 & 164 & 20.6 & 9,0 & 140 & 17.6 & 7,7 & 33 & 4.2 & 795 & 78,9 & 43,8 \\
\hline & Feminino & 126 & 59.2 & 6,1 & 5 & 2.3 & 0,2 & 31 & 14.6 & 1,5 & 40 & 18.8 & 1,9 & 11 & 5.2 & 213 & 21,1 & 10,3 \\
\hline & Subtotal & 533 & 52.9 & - & 56 & 5.6 & - & 195 & 19.3 & - & 180 & 17.9 & - & 44 & 4.4 & 1008 & 100,0 & - \\
\hline & Ignorado & 13 & 54.2 & - & 0 & 0.0 & - & 4 & 16.7 & - & 3 & 12.5 & - & 4 & 16.7 & 24 & - & - \\
\hline & Total & 546 & 52.9 & 14,1 & 56 & 5.4 & 1,4 & 199 & 19.3 & 5,1 & 183 & 17.7 & 4,7 & 48 & 4.7 & 1032 & 100,0 & 26,6 \\
\hline \multirow{8}{*}{ 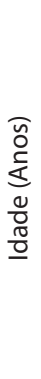 } & $0-9$ & 8 & 47.1 & 1,2 & 3 & 17.6 & 0,5 & 2 & 11.8 & 0,3 & 3 & 17.6 & 0,5 & 1 & 5.9 & 17 & 1,7 & 2,6 \\
\hline & $10-19$ & 59 & 58.4 & 7,7 & 7 & 6.9 & 0,9 & 17 & 16.8 & 2,2 & 13 & 12.9 & 1,7 & 5 & 5.0 & 101 & 10,3 & 13,2 \\
\hline & $20-39$ & 306 & 47.8 & 22,7 & 28 & 4.4 & 2,1 & 156 & 24.4 & 11,6 & 130 & 20.3 & 9,7 & 20 & 3.1 & 640 & 65,0 & 47,5 \\
\hline & $40-59$ & 110 & 63.6 & 14,6 & 12 & 6.9 & 1,6 & 18 & 10.4 & 2,4 & 26 & 15.0 & 3,5 & 7 & 4.0 & 173 & 17,6 & 23,0 \\
\hline & 60 e mais & 42 & 77.8 & 12,1 & 2 & 3.7 & 0,6 & 1 & 1.9 & 0,3 & 6 & 11.1 & 1,7 & 3 & 5.6 & 54 & 5,5 & 15,6 \\
\hline & Subtotal & 525 & 53.3 & - & 52 & 5.3 & - & 194 & 19.7 & - & 178 & 18.1 & - & 36 & 3.7 & 985 & 100,0 & - \\
\hline & Ignorado & 21 & 44.7 & - & 4 & 8.5 & - & 5 & 10.6 & - & 5 & 10.6 & - & 12 & 25.5 & 47 & - & - \\
\hline & Total & 546 & 52.9 & 14,1 & 56 & 5.4 & 1,4 & 199 & 19.3 & 5,1 & 183 & 17.7 & 4,7 & 48 & 4.7 & 1032 & 100,0 & 26,6 \\
\hline
\end{tabular}

(1) TI: Taxa de incidência (Número de ocorrências/ população exposta* 10 mil habitantes) / TI: Incidence rate (Number of events/population exposed* 10,000 inhabitants) 
Tabela 2 - Distribuição absoluta e relativa, incidência e risco relativo de atendimentos do Samu por ATT, segundo mês, dia da semana e horário da ocorrência. Olinda, PE - Junho de 2006 a Julho de 2007.

Table 2 - Absolute and relative distribution, incidence and relative risk of care of the mobile emergency care per accident according to month, day of they week and time of occurrence; Olinda, PE, Brazil; July 2006 to June 2007.

\begin{tabular}{|c|c|c|c|c|c|c|c|c|}
\hline & & Período & $\begin{array}{c}\mathrm{N} \text { de } \\
\text { atendimentos }\end{array}$ & $\%$ & $\mathrm{TI}^{(1)}$ & $\mathbf{R R}^{(2)}$ & IC 95\% ${ }^{(3)}$ & p-Valor \\
\hline \multirow{7}{*}{ 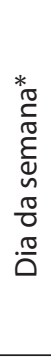 } & & $2^{\text {a feira }}$ & 133 & 12,9 & 3,4 & 1,5 & $(1,1-1,3)$ & 0,004 \\
\hline & & $3^{a}$ feira & 118 & 11,4 & 3,0 & 1,3 & $(1,0-1,7)$ & 0,052 \\
\hline & & $4^{\text {a feira }}$ & 90 & 8,7 & 2,3 & 1,0 & & \\
\hline & & $5^{\text {a feira }}$ & 110 & 10,7 & 2,8 & 1,2 & $(0,9-1,6)$ & 0,157 \\
\hline & & $6^{a}$ feira & 146 & 14,1 & 3,8 & 1,6 & $(1,2-2,1)$ & 0,000 \\
\hline & & Sabado & 213 & 20,6 & 5,5 & 2,4 & $(1,8-3,3)$ & 0,000 \\
\hline & & Domingo & 222 & 21,5 & 5,7 & 2,5 & $(1,9-3,2)$ & 0,000 \\
\hline \multirow{8}{*}{ 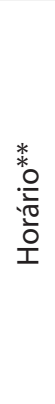 } & \multirow{4}{*}{ 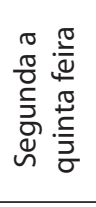 } & $00 \mathrm{~h} 00 \mathrm{~min}-05 \mathrm{~h} 59 \mathrm{~min}$ & 40 & 9,4 & 1,0 & 1,0 & & \\
\hline & & 06h00min - 11h59min & 99 & 23,3 & 2,6 & 2,5 & $(1,7-3,6)$ & 0,000 \\
\hline & & $12 \mathrm{~h} 00 \mathrm{~min}-17 \mathrm{~h} 59 \mathrm{~min}$ & 124 & 29,2 & 3,2 & 3,1 & $(2,2-4,4)$ & 0,000 \\
\hline & & 18h00min -23h59min & 161 & 38,0 & 4,2 & 4,0 & $(2,8-5,7)$ & 0,000 \\
\hline & \multirow{4}{*}{ 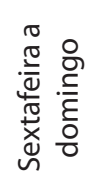 } & 00h00min - 05h59min & 88 & 16,3 & 2,3 & 1,0 & & \\
\hline & & 06h00min - 11h59min & 102 & 18,9 & 2,6 & 1,2 & $(0,9-1,5)$ & 0,310 \\
\hline & & $12 \mathrm{~h} 00 \mathrm{~min}-17 \mathrm{~h} 59 \mathrm{~min}$ & 129 & 23,9 & 3,3 & 1,5 & $(1,1-1,9)$ & 0,005 \\
\hline & & 18h00min -23h59min & 221 & 40,9 & 5,7 & 2,5 & $(2,0-3,2)$ & 0,000 \\
\hline
\end{tabular}

(1)TI:Taxa de incidência (Número de ocorrências/ população exposta* 10 mil habitantes)

(2) RR = Incidência esperada/ Incidência observada Cl: Coeficiente de incidência (casos/10 mil habitantes)

(3) IC: Intervalo com $95 \%$ de confiança

*Considerando como dia de referência a 4 a feira

** Considerando como horário de referência 00h00min às $05 \mathrm{~h} 59 \mathrm{~min} ; 68$ formulários tiveram horário de atendimento ignorado.

(1) TI: Incidence rate (Number of events/ population exposed* 10,000 inhabitants)

(2) $R R=$ Expected Incidence/Observed Incidence of Cl: Incidence Coefficient (cases/10,000 inhabitants)

(3) IC: $95 \%$ confidence interval

*Considering Wednesday as reference day

** Considering 00h00min to 05 h59min as reference time; 68 forms did not report time of care.

vítima. As motocicletas foram as principais causadoras de atropelamentos (235; 43,0\%), seguidas dos carros de passeio $(108 ; 19,8 \%)$ e dos ônibus (87; 15,9\%).

\section{Distribuição espacial dos atendimentos por acidentes de transportes terrestres realizados pelo Samu-192 Olinda}

As áreas críticas para atendimentos por ATT, indicadas pelo Índice de Moran em sua terceira etapa, representada pelo moranmap, estão apresentadas na Figura 3; as características dessas regiões são descritas a seguir:

- Giradouro de Olinda: Principal entrada do município, que liga três eixos viários da cidade. Destacam-se nessa região os acidentes que têm como vítimas os ocupantes de outros veículos, pedestres e ciclistas.

- Terminal Integrado da PE 15: O Terminal Integrado da PE 15 atende passageiros e recebe veículos vindos da Região Metropolitana Norte, da II ${ }^{a}$ Perimetral e da Av. Bultrins, apresentando corredores de ônibus e vias bem conservadas.

- Corredor da orla litorânea: Passagem obrigatória para aqueles que saem das praias de Olinda; a área tem também grande concentração de bares noturnos.

- Av. II Perimetral: Avenida que liga a Rodovia PE-15 à Av. Pres. Kennedy privilegia o trânsito de veículos, tendo pouco espaço para a circulação de pedestres. É permeada, em parte, pelo 


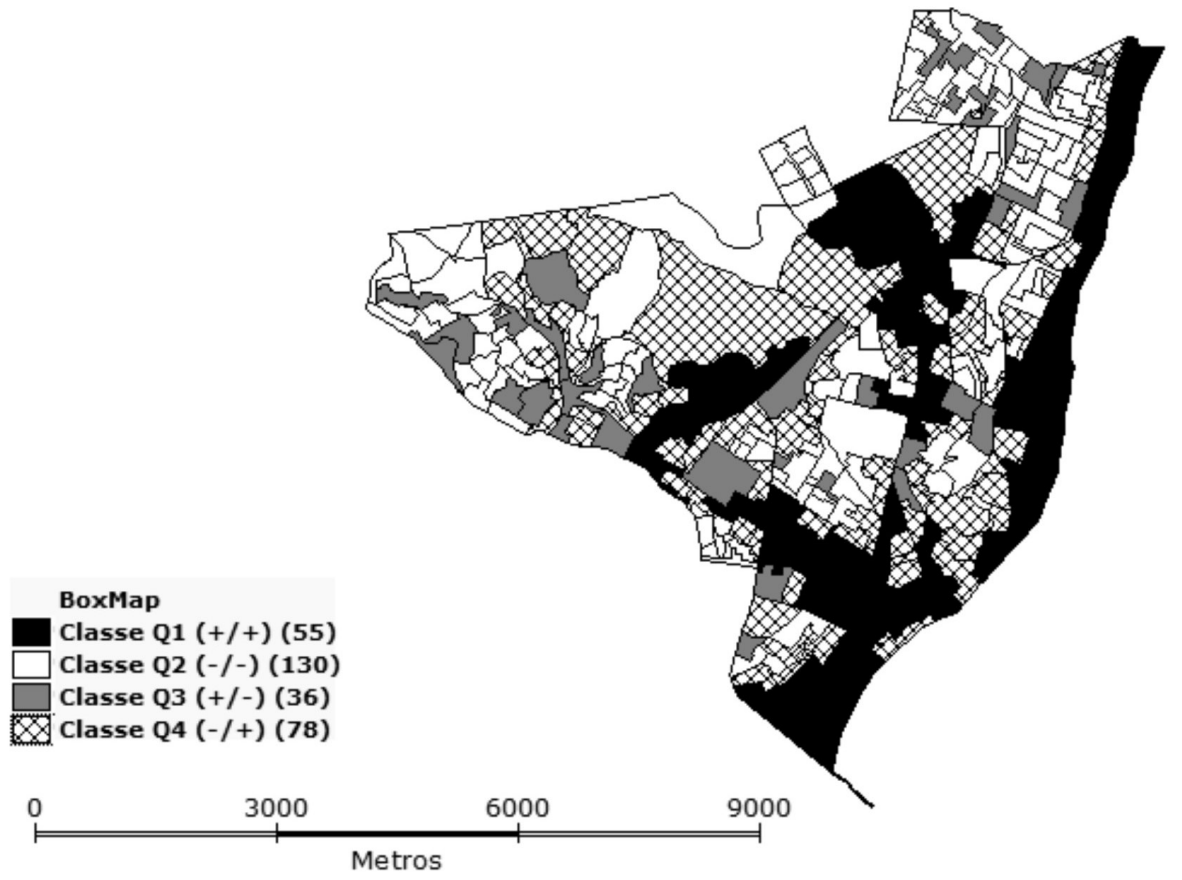

Figura 1 - Áreas críticas para atendimentos por acidentes de transportes terrestres (Boxmap). Olinda, PE - Junho de 2006 a Julho de 2007.

Figure 1 - Critical areas for mobile emergency care due to land transportation accidents (Boxmap); Olinda, PE, Brazil; July 2006 to June 2007.

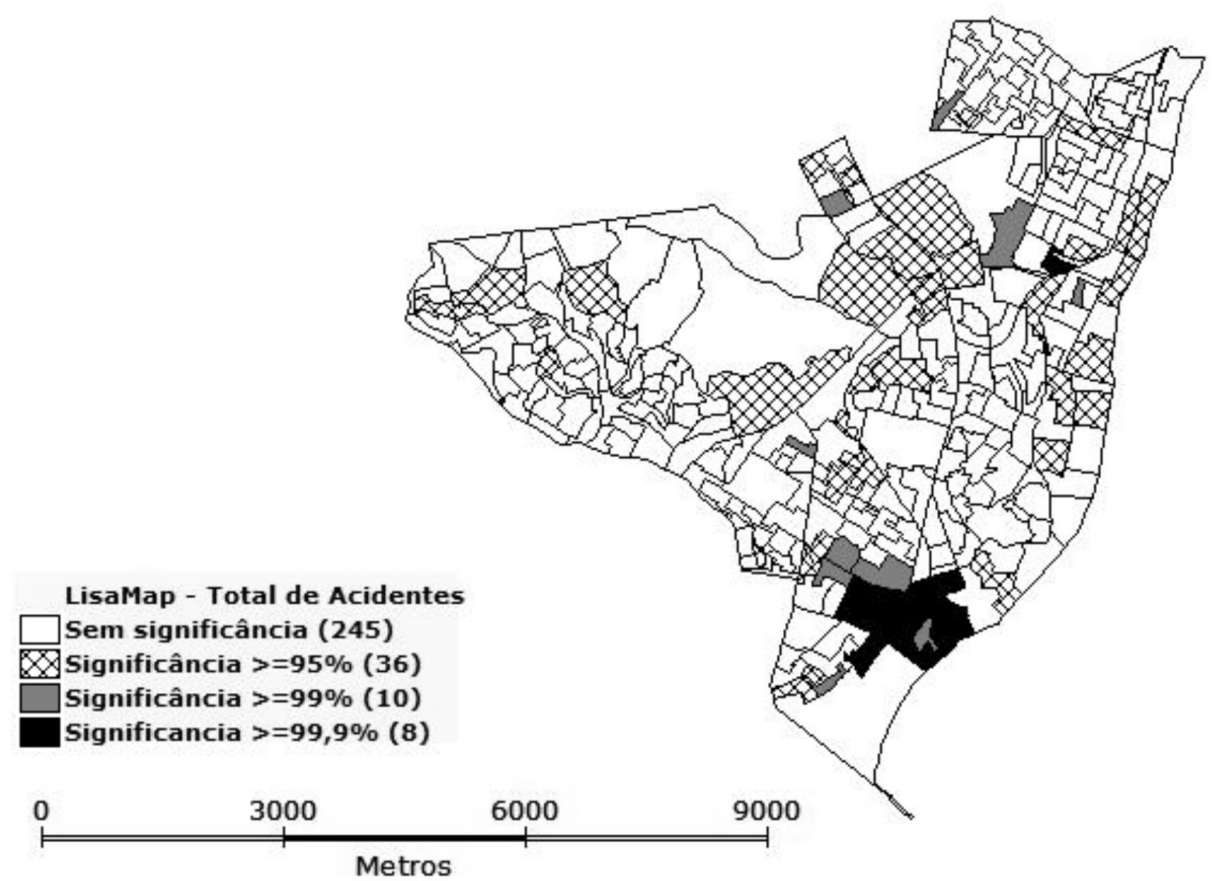

Figura 2 - Áreas críticas para atendimentos por acidentes de transportes terrestres (Lisamap). Olinda, PE - Junho de 2006 a Julho de 2007.

Figure 2 - Critical areas for mobile emergency care due to land transportation accidents (Lisamap); Olinda, PE, Brazil; July 2006 to June 2007. 


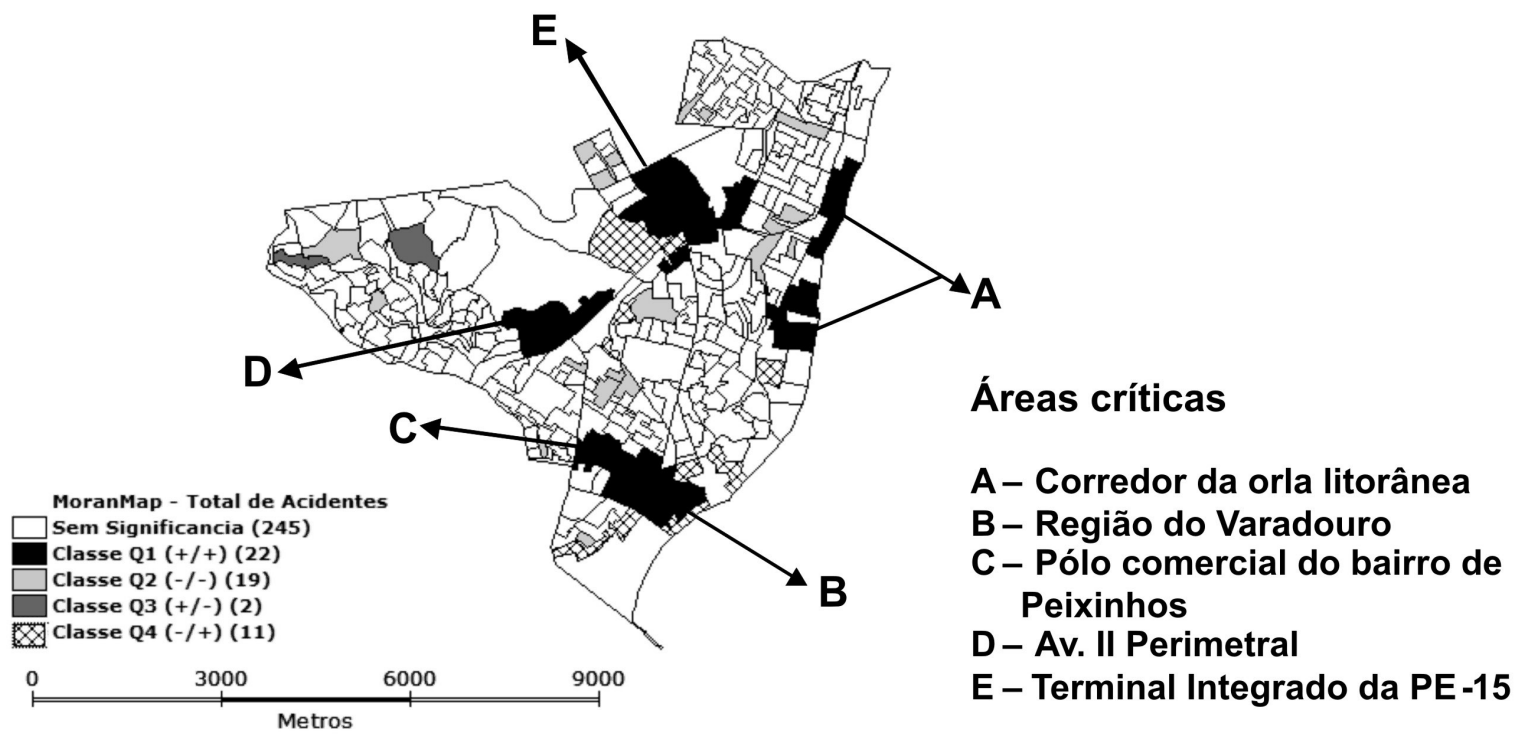

Figura 3 - Áreas críticas para atendimentos por acidentes de transportes terrestres (Moranmap). Olinda, PE - Junho de 2006 a Julho de 2007.

Figure 3 - Critical areas for mobile emergency care due to land transportation accidents (Moranmap); Olinda, PE, Brazil; July 2006 to June 2007.

Aterro Controlado de Aguazinha, que atrai diariamente aqueles que trabalham com recicláveis (sobretudo a partir das 18 horas) e pessoas que dependem de alguma forma dos resíduos ali depositados. Possui iluminação e sinalização deficientes, o que a torna arriscada tanto para pedestres como para ocupantes de veículos.

- Pólo comercial do bairro de Peixinhos: Região de destaque para os atropelamentos, concentra lojas, feira livre e tem em suas imediações escolas e estabelecimentos de saúde, atraindo grande número de pedestres de todos os sexos e faixas etárias. A principal avenida da região, a Presidente Kennedy, concentra grande fluxo de ônibus e demais veículos da zona oeste da cidade, limítrofe à Cidade do Recife. O calçamento irregular e o comércio ambulante são características locais que também podem contribuir para que, muitas vezes, 0 pedestre passe a usar as áreas destinadas exclusivamente aos veículos, gerando uma maior exposição deste grupo aos ATT.

- Região do Varadouro: É uma opção de acesso ao Centro Histórico de Olinda e à região litorânea. Apresenta grande fluxo de veículos e os pedestres foram as maiores vítimas de ATT nessa região. Destaca-se a presença de uma escola privada na região crítica, o que alerta a necessidade da mobilização de diversos setores da sociedade na minimização destes eventos.

\section{Discussão}

O fato de aproximadamente $80,0 \%$ dos atendimentos serem destinados a pedestres, ciclistas e ocupantes de motocicleta mostra a vulnerabilidade destes grupos de vítimas, pois no impacto dos acidentes o choque desigual com veículos de maior porte é bastante frequente.

Os pedestres concentraram mais da metade dos atendimentos. Na cidade do Rio de Janeiro, uma análise de morbidade hospitalar por acidentes de trânsito em hospitais públicos constatou que $49,4 \%$ dos pacientes eram pedestres, sendo estes os que apresentaram maior média de permanência e maior taxa de letalidade por causas externas no setor de emergência ${ }^{21}$. 
Essas informações alertam para a importância de uma equipe de resgate treinada e experiente, visando impedir o agravamento do quadro e proporcionar uma maior sobrevida à vítima, mantendo-a em condições de chegar com vida até que o tratamento hospitalar seja possível.

As ações voltadas para a organização das vias públicas e o seu tráfego têm privilegiado o trânsito dos veículos motorizados, deixando os pedestres em situação de desvantagem em relação aos motoristas e passageiros, e gerando um elevado número de atropelamentos ${ }^{22}$. Esforços para mudar o comportamento dos pedestres por meio da educação também são importantes, mas surtem menos efeito do que ações que tenham como objetivo modificar o ambiente físico e social do sistema de transportes ${ }^{23}$.

Os motociclistas se destacaram neste estudo como vítimas e causadores de acidentes. Esse grupo é mais vulnerável quando comparado aos usuários de outros veículos automotores. Por outro lado, cabe a reflexão de que os motociclistas comumente colocam em risco a integridade de outros usuários, principalmente pedestres ${ }^{23}$. A motocicleta se tornou viável no trânsito lento e congestionado, sendo econômica, de custo bem inferior quando comparada aos automóveis, de fácil aquisição e manejo. Apesar da frota de automóveis ser superior à frota de motocicletas, essas facilidades têm levado a uma inversão nos padrões de acidentes de trânsito: as vítimas de acidentes de trânsito envolvendo motocicletas (motociclistas e pedestres) predominam em relação àquelas envolvendo os ocupantes de automóveis ${ }^{23,29}$.

Aproximadamente 19\% das vítimas eram ocupantes de veículos do tipo carro de passeio, caminhão e/ou ônibus. Provavelmente, a proteção conferida aos ocupantes do próprio veículo ou o uso do cinto de segurança pode estar favorecendo esse baixo percentual $^{23}$.

$\mathrm{O}$ fato de apenas 56 atendimentos do Samu 192 Olinda terem se destinado aos ciclistas vítimas de ATT não minimiza a gravidade do evento. Há relatos de sub- registros de acidentes com lesão corporal envolvendo ciclistas, sendo muitas vezes classificados como pedestres ${ }^{24}$. Em Pelotas, Rio Grande do Sul, uma comparação entre boletins de ocorrência e atendimentos no pronto-socorro, durante dois anos, revelou $33 \%$ de sub-registro dos acidentes envolvendo ciclistas $^{23}$.

A ausência de equipamentos de segurança da bicicleta e sua utilização como meio de transporte para deslocamento ao trabalho são duas questões que precisam ser consideradas na análise dos acidentes que envolvem ciclistas. O estudo Planejamento Cicloviário: Diagnóstico Nacional, que contou com a participação de sessenta municípios brasileiros, revelou que quase dois terços da frota de bicicletas são utilizados como meio de transporte da classe operária, sendo a renda apontada como fator preponderante para a escolha da bicicleta, em se tratando de deslocamento para o trabalho ${ }^{25}$. Entretanto, o desconhecimento e o baixo poder aquisitivo podem impossibilitar a manutenção da bicicleta em boas condições ${ }^{24}$. Um estudo realizado com 293 trabalhadores que utilizavam bicicletas para locomoção em na cidade de Pelotas, no ano de 2003, revelou que $15 \%$ das bicicletas não tinham freios, $20 \%$ não tinham refletor de pedais e apenas $5 \%$ contavam com espelho retrovisor ${ }^{24}$. Aliadas aos problemas já expostos, a infra-estrutura cicloviária insuficiente e a baixa conscientização de motoristas de veículos automotores para a presença de ciclistas em via pública contribuem para o número considerável de acidentes de trânsito envolvendo ciclistas.

$\mathrm{O}$ fato de homens e jovens serem as vítimas mais frequentes no trânsito se assemelha a diversos estudos ${ }^{7,23,26,27}$. Muito provavelmente, esse perfil é consequência da maior exposição masculina e de jovens no trânsito, e de comportamentos determinados social e culturalmente, que os fazem assumir maiores riscos na condução de veículos, como maior velocidade, manobras mais arriscadas e uso de álcool. É possível, entretanto, que esse quadro se altere num futuro próximo, por estar ocorrendo uma 
clara tendência de aumento da participação feminina na condução de veículos automotores, devido à sua crescente inserção no mercado de trabalho, maior independência e consequente maior exposição ao tráfego das cidades.

Quanto ao mês de atendimento, seria interessante analisar o comportamento dessa variável num maior período de tempo, pois diversos fatores em um único ano podem influenciar a proporção de atendimentos/mês. O mês de fevereiro é peculiar em Olinda, devido às festas carnavalescas, que levam um maior número de pessoas às ruas, ampliando a exposição da população aos acidentes de transportes terrestres.

Semelhante ao observado em outros estudos $^{5,8,27}$, houve predominância de atendimentos entre $18 \mathrm{~h} 00$ e 23h59. Além da elevação do fluxo de veículos, comum no retorno da escola ou do trabalho, possivelmente os acidentes ocorridos neste horário podem ser explicados pelo acúmulo de cansaço, exigências e preocupações que acabam por desgastar o físico e mente das pessoas, deixando-as mais vulneráveis aos acidentes.

Nos finais de semana e à noite é mais provável o consumo de bebidas alcoólicas, o que aumenta o risco de acidentes. A diminuição da visibilidade no período noturno, o menor tráfego e o abuso da velocidade são outros fatores que aumentam o número de acidentes de maior gravidade $\mathrm{e}^{5,23,28,29}$.

Embora os ônibus estejam presentes em apenas $9,7 \%$ do total de ocorrências, chama atenção o fato de $15,9 \%$ de os atendimentos aos pedestres terem sido motivado por choques com ônibus. É necessária uma reavaliação das políticas públicas atuais, que pouco enfatizam a melhoria do ambiente para a circulação mais segura e eficiente de pedestres e outros tipos de veículos, como bicicletas e ônibus de transporte coletivo público ${ }^{7,23}$.

O alto percentual de motocicletas envolvidas nos acidentes analisados no presente estudo, além do volume de ocupantes deste veículo vitimados no período, como discutido anteriormente, reforça a necessidade do trabalho de educação no transito específico para este público, uma vez que essa frota tem crescido mais que a de outros veículos automotores $^{6}$. No ano de 2001, a proporção de motocicletas era de $14,4 \%$ do total dos veículos automotores do país, enquanto em 2005 essa proporção atingiu 19,4\% .

Utilizar os setores censitários permitiu identificar regiões de maiores riscos de atendimentos por ATT, inclusive com significância estatística, o que para a gestão se traduz em áreas prioritárias de intervenção. A ocorrência do ATT muitas vezes é determinada pelo cenário em torno das vias, como a ausência ou deficiência no calçamento, gerando disputa pelo espaço entre pedestres e veículos, como observado na Av. II Perimetral; e também pela presença do comércio ou de equipamentos urbanos, provocando maior concentração de pessoas, como no Pólo Comercial de Peixinhos.

O eixo viário da região do Giradouro de Olinda atualmente passa por reformas, com previsão de conclusão no ano de 2009. Por outro lado, as boas condições da região do Terminal integrado da PE 15 estimulam o excesso de velocidade, comportamento também observado no Corredor da orla litorânea - nesse trecho também associado ao uso de bebidas alcoólicas. Além das mudanças estruturais, devem ser incentivadas atitudes corretas dos pedestres, ciclistas e condutores de veículos em geral para a promoção da cultura de paz no trânsito.

\section{Conclusões}

Trabalhar com o local de ocorrência do acidente, em vez de residência das vítimas, permite maior utilidade das informações para as áreas de assistência à saúde, bem como o planejamento e a administração do tráfego. $\mathrm{O}$ uso da análise espacial mostra o quanto é fundamental a integração entre a Secretaria Municipal de Saúde de Olinda e órgãos afins para a implantação e implementação de medidas preventivas e corretivas.

A partir dos achados deste estudo, conclui-se que a fragilidade e o volume de 
pedestres e motociclistas vítimas de ATT reforçam argumentos já intensamente colocados sobre a necessidade de intervenções amplas, intersetoriais e multiprofissionais, que promovam um ambiente mais favorável para a circulação dessas pessoas.

O predomínio do sexo masculino e de adultos jovens mostra que são oportunos programas de educação para o trânsito, com técnicas adequadas, visando atingir esse grupo de risco. Em relação aos dias e horários de maior número de atendimentos por ATT no município de Olinda, a fiscalização por parte dos órgãos de trânsito poderia ser intensificada a partir das 18 horas, sobretudo nos finais de semana.

Os motociclistas e seus veículos se destacaram por estarem envolvidos em mais da metade das ocorrências atendidas. Além do uso para fins de lazer, muitos destes condutores estão em situações de trabalho. É necessária a integração com empresas que se utilizam desses serviços com representantes da categoria profissional envolvida, bem como a busca e execução de atividades intersetoriais para a melhoria da segurança no tráfego e a redução desses acidentes. $\mathrm{O}$ setor saúde pode ser útil contribuindo com atividades educativas direcionadas para este grupo, além de promover pesquisas para compreender o fenômeno. Parcerias também são necessárias com as empresas de transporte coletivo, haja vista a partici- pação expressiva de ônibus em acidentes, sobretudo vitimando pedestres.

Este estudo ratifica o importante papel do Sistema de Informação Geográfica e da análise espacial na vigilância dos ATT, pois um conjunto de informações pode ser utilizado para o monitoramento das áreas alvo de intervenção, seja nos principais eixos viários, seja na organização do espaço urbano. A construção de um banco de dados que permita extrair informações sobre vítimas de ATT na fase pré-hospitalar em nível local possibilita o monitoramento permanente destes eventos, ao identificar áreas geográficas e fatores associados ao maior risco de atendimentos no município. É pertinente estimular o uso dessa fonte de dados, incentivar a completitude do formulário de atendimento, trabalhar no aprimoramento do banco de dados e divulgar seus dados aos gestores, profissionais de saúde e o público em geral.

Agradecimentos: Os autores agradecem a Secretaria Municipal de Saúde de Olinda, em especial ao Serviço de Atendimento Móvel de Urgência (Samu Olinda), Diretoria de Vigilância em Saúde, Diretoria de Planejamento em Saúde, Núcleo de Prevenção a Acidentes e Violência e Núcleo de Geoprocessamento pelo apoio logístico que possibilitou a execução deste trabalho.

\section{Referências}

1. World Health Organization. World report on Road traffic injury prevention. Geneva: WHO; 2004. Disponível em: http://www.who.int/violence_injury_prevention/ publications/road_traffic/world_report/en/index.html. [Acessado em 2 de janeiro de 2008].

2. Souza ER, Minayo MC, Malaquias JV. Violência no transito: expressão da violência social in Impacto da Violência na saúde dos brasileiros, série B. Textos Básicos de saúde-Brasília/DF; 2005.

3. Souza MFM, Malta DC, Conceição GMS et al. Análise descritiva e de tendência de acidentes de transporte terrestre para políticas sociais no Brasil. Epidemiol Serv Saúde 2007; 16(1); 33-44. ISSN 1679-4974.

4. Belo Horizonte. Secretaria Municipal de Saúde. Pesquisa de acompanhamento de vítimas de acidentes de trânsito em Belo Horizonte, 2005. Disponível em http:// dtr2001.saude.gov.br/samu/artigos/saude_transito.htm. [Acessado em 10 de julho de 2006].

5. Soares DFPP, Barros MBA. Fatores associados ao risco de internação por acidentes de trânsito no Município de Maringá-PR. Rev Bras Epidemiol 2006; 9(2): 193-205.

6. Mello Jorge MHP, Koizumi MS. Acidentes de trânsito no Brasil: Um atlas de sua distribuição. São Paulo: Abramet; 2007.

7. Andrade SM, Mello Jorge MHP. Características das vítimas por acidentes de transporte terrestre em município da Região Sul do Brasil. Rev Saúde Pública 2000; 34: 149-56. 
8. Andrade SM, Mello Jorge MHP. Acidentes de transporte terrestre em cidade da Região Sul do Brasil: Avaliação da cobertura e qualidade dos dados. Cad Saúde Pública 2001; 17: 1449-56.

9. Rede Interagencial de Informações para a Saúde. Indicadores básicos para a saúde no Brasil: conceitos e aplicações; 2008.

10. Gawryszewski VP et al. A proposta da rede de serviços sentinela como estratégia da vigilância de violências e acidentes. Ciênc Saúde Coletiva 2006; 11(S): 1269-78. ISSN 1413-8123.

11. Cabral APS, Souza WV. Serviço de Atendimento Móvel de Urgência (SAMU): análise da demanda e sua distribuição espacial em uma cidade do Nordeste brasileiro. Rev Bras Epidemiol 2008; 11(4): 530-40. ISSN 1415-790X

12. Ministério da Saúde, Secretaria de Vigilância em Saúde. Mortalidade por acidentes de transporte terrestre no Brasil. In: Saúde Brasil 2006 Uma análise da desigualdade em saúde, Série G. Estatística e Informação em Saúde. Brasília; 2007.

13. Brasil. Portaria No 1.863 /GM de 29 de setembro de 2003. Diário Oficial da República Federativa do Brasil, Brasília DF, 6 de outubro de 2003. Disponível em http:/ / www. cremesp.org.br/library/modulos/legislacao/versao_ impressao.php?id=3232. [Acessado em 10 de maio de 2006].

14. Brasil. Portaria No 2048/GM de 5 de novembro de 2002. Diário Oficial da República Federativa do Brasil, Brasília, DF, 12 de novembro de 2002. Disponível em http:// dtr2001.saude.gov.br/samu/legislacao/leg_2048.htm. [Acessado em 10 de maio de 2006].

15. Brasil. Ministério da Saúde. SAMU-192: O que é o $S A M U$ ? Disponível em http://www.saude.gov.br/samuprograma-nacional.htm. [Acessado em 7 de junho de 2006].

16. Secretaria da Saúde de Estado de Goiás. Urgência e emergência. Disponível em http:/ / www.saude.go.gov.br/ index.php?idMateria=6240. [Acessado em 1 de março de 2009].

17. Brasil. Ministério da Saúde. Departamento de Informática do SUS. Informações de Saúde. Demográficas e socioeconômicas. Disponível em http:// www.datasus.gov.br. [Acessado em 15 de janeiro de 2007].

18. Brasil. Agência Nacional das Águas. Relatório de Conjuntura de Recursos Hídricos 2005. Disponível em http://www.ana.gov.br/portalestudos/ conjuntura/arquivosparadowload/ nivelPais/ DensidadeDemograficaPorMunicipioEEstados.pdf. [Acessado em 5 de novembro de 2007].
19. Anselin L. Local indicators of spatial association - LISA. Geographical Analysis 1995; 27(2): 93-115.

20. Santos L, Raia Junior AA. Análise Espacial de Dados Geográficos: A Utilização da Exploratory Spatial Data Analysis - ESDA para Identificação de Áreas Críticas de Acidentes de Trânsito no Município de São Carlos (SP). Sociedade \& Natureza 2006; 18 (35): 97-107.

21. Deslandes SF, Silva CMFP. Análise da morbidade hospitalar por acidentes de trânsito em hospitais públicos do Rio de Janeiro, RJ, Brasil. Rev Saúde Pública [online] 2000; 34(4): 367-72. ISSN 0034-8910.

22. Faria EO, Braga MGC. Propostas para minimizar os riscos de acidentes de trânsito envolvendo crianças e adolescentes. Ciênc Saúde Coletiva [online] 1999; 4(1): 95-107. ISSN1413-8123.

23. Bastos YGL, Andrade SM, Soares DA. Características dos acidentes de trânsito e das vítimas atendidas em serviço pré-hospitalar em cidade do Sul do Brasil, 1997/2000. Cad Saúde Pública [online] 2005; 21(3): 815-22. ISSN 0102-311X

24. Bacchieri G, Gigante DP, Assuncao, MC. Determinantes e padrões de utilização da bicicleta e acidentes de trânsito sofridos por ciclistas trabalhadores da cidade de Pelotas, Rio Grande do Sul, Brasil. Cad Saúde Pública [online] 2005; 21(5): 1499-508. ISSN 0102-311X.

25. Empresa Brasileira de Planejamento de Transportes. Planejamento cicloviário: diagnóstico nacional. Brasília: Empresa Brasileira de Planejamento de Transportes; 2001.

26. Malvestio MAA, Sousa RMC. Suporte avançado à vida: Análise do atendimento a vítimas de acidentes de trânsito. In: XV SBAIT, 2002, São Paulo. XV SBAIT; 2002.

27. Oliveira NLB, Sousa RMC. Retorno à atividade produtiva de motociclistas vítimas de acidentes de trânsito. Acta Paul Enferm 2006; 19(3): 284-9.

28. Santos MR. Vítimas do trânsito em São José do Rio Preto [tese de doutorado]. Faculdade de Medicina de São José do Rio Preto. Área de concentração: Ciências da Saúde; 2004.

29. Oliveira NLB. Fatores associados ao risco de lesões e óbito de motociclistas envolvidos em ocorrência de trânsito [tese de doutorado]. Universidade de São Paulo: São Paulo; 2008. Disponível em http:/ / www.teses.usp. br/teses/disponiveis/7/7139/tde-11032008-111333. [Acessado em 1 de março de 2009].

Recebido em: 27/10/09 Versão final reapresentada em: 04/09/10 Aprovado em: 04/10/10 